\title{
Transparent conducting film: Effect of mechanical stretching to optical and electrical properties of carbon nanotube mat
}

\author{
TSUYOSHI SAOTOME*, HANSANG KIM, DAVID LASHMORE ${ }^{\dagger}$ and \\ H THOMAS HAHN ${ }^{\dagger \dagger}$ \\ UCLA Mechanical and Aerospace Engineering Department, Multifunctional Composites Laboratory, \\ Los Angeles CA 90095, USA \\ ${ }^{\dagger}$ Nanocomp Technologies, Inc, 162 Pembroke Road, Concord, NH 03301 \\ ${ }^{\dagger \dagger}$ California NanoSystems Institute, 570 Westwood Plaza, Building 114, Los Angeles CA 90095, USA
}

MS received 26 July 2009; revised 19 October 2009

\begin{abstract}
We describe in this paper a transparent conducting film (TCF). It is a fibrous layer of multiwalled carbon nanotubes (MWNTs), labeled a dilute CNT mat, that was prepared and unidirectionally stretched to improve both the optical and electrical properties. After stretching by $80 \%$ strain, transmittance at $550 \mathrm{~nm}$ wavelength was improved by $37 \%$ and sheet resistance was reduced to $71 \%$ of the original value. The improvement of the transmittance can be explained by increased area of the CNT mat after stretch, and the reduced sheet resistance can be explained by increased density of the CNT alignment in lateral direction due to contraction. Based on the microscopic observation before and after stretch, models to describe the phenomena are proposed. By further expanding on this method, it may be possible to obtain a transparent conducting carbon nanotube film which is crack-resistant for solar cell applications.
\end{abstract}

Keywords.

\section{Introduction}

Solar-powered unmanned aerial vehicles (UAV) have been studied for environmental monitoring, weather forecasting, high altitude communications, and military reconnaissance purposes (Grace et al 2001; Teets et al 2002; MacDonald 2005). Current commercial solar cells widely use indium tin oxide (ITO) as a transparent conducting film to transport the excited electrons. However, ITO's inherent fragility causes degradation of electric conductivity (Cairns et al 2005) especially when considering large structural deformations and vibrations in solar UAV applications. Carbon nanotubes (CNTs) (Iijima 1991) with strong fibrous networks are expected to overcome the fragility issue, and thus widely studied for transparent conducting film (TCF) applications (Hu et al 2004; Wu et al 2004; Zhang et al 2005; Zhou et al 2006). A study claims that CNT film shows an excellent ability to hold its electrical conductivity under bending, which could not be achieved by conventional ITO (Zhang et al 2005).

In this study, a low-density (dilute) fibrous layer of multi-walled carbon nanotubes (MWNTs), labeled a dilute CNT mat, was prepared and unidirectionally stretched to examine how this elongation would affect

\footnotetext{
*Author for correspondence (saotome@naa.att.ne.jp)
}

both optical and electrical properties. Due to the area increase after stretch, the density of the dilute CNT mat was reduced and resulted in improved visible light transmittance. Since the dilute CNT mat was stretched unidirectionally, lateral MWNT contraction occurred, resulting in reduced sheet resistance. Both phenomena can be explained by a simple model and will be discussed in more details.

Once the dilute CNT mat, which was temporarily supported by a substrate film to avoid the breakage (detailed in section two), is successfully stretched, it can be transferred onto a transparent protective film with a thin adhesive layer on one side. For example, PET film with a transparent adhesive, produced by Teraoka Seisakusho Co. Ltd (Tokyo, Japan), can be used for the purpose. Since the stiffness of the dilute CNT mat is found to be much lower than that of the protective layer from our experiences, residual stress remained on the protective layer by the stretched dilute CNT mat after the transplantation is considered to be negligible. The composite of the stretched dilute CNT mat/transparent protective layer is then directly used for the photovoltaic application.

The dilute CNT mat shows tremendous potential as the transparent conducting element in solar cells. Since the dilute CNT mat can be fabricated without using the vacuum filtration method, it can be manufactured in much larger areas without the limitations of filter dimension present when using the vacuum filtration method 
(Hu et al 2004; Zhou et al 2006). This shows great promise in scalability to large-scale fabrication of transparent conducting CNT films.

\section{Experimental}

A dilute CNT mat which had original visible light transmittance of $50-60 \%$ at $550 \mathrm{~nm}$ wavelength was prepared by our partner, Nanocomp Technologies, Inc. In its fabrication process, MWNTs synthesized by a typical chemical vapour deposition method were collected on a rolling conveyer, and the loosely collected MWNTs were pressed to form a sheet like structure (Lashmore et al 2007). Visual inspection of the dilute CNT mat showed non-uniform distribution of the CNT network (figure 1). Presented in figure 2 are scanning electron microscopy (SEM) images of a dilute CNT mat, which showed both areas with well-dispersed MWNTs and areas with bundled MWNTs. Bundles are defined as clusters of MWNTs attracted to each other by van der Waals forces. The average size of the bundles was measured to be approximately hundreds of nanometres to a few micrometres in width.

The effect of further dilution or thinning of the MWNT density on visible light transmittance and sheet resistance was studied by unidirectionally stretching the CNT mat with the help of a polymer substrate film. The CNT mat was placed on two different substrate films: one with adhesive (1867T12 polyurethane elastomer. 3M corp., St. Paul MN, $t=0.305 \mathrm{~mm}$ ), and one without (polyvinyl chloride, Polyvinyl Films, Inc., Sutton MA, $t=0.011$ $\mathrm{mm})$. The film with attached CNT mat was unidirectionally stretched by various amounts. The polyurethane elastomer film had an adhesive on one side to hold the CNT mat. The polyvinyl chloride film did not. The dilute CNT mat is thought to adhere to the polyvinyl chloride film by

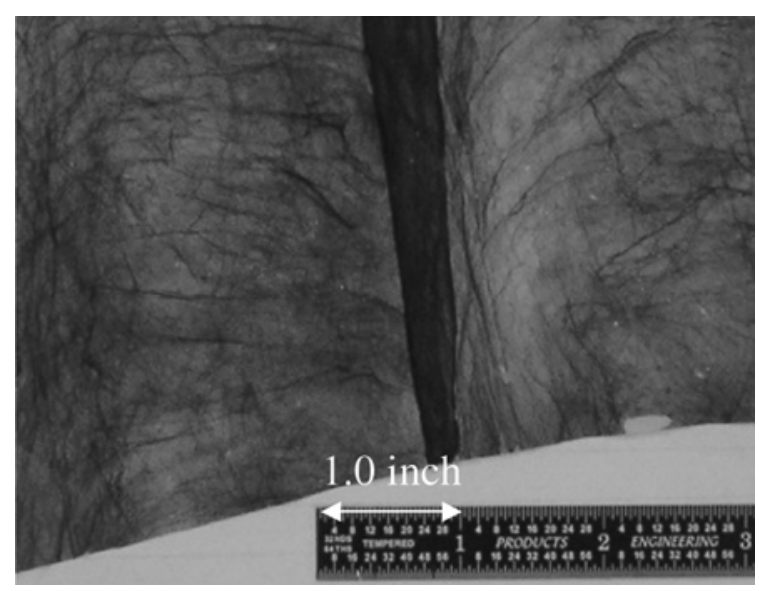

Figure 1. A dilute CNT mat prepared by Nanocomp Technologies, Inc. Non-uniform distribution of CNT network could be observed by visual inspection. van der Waals force. Van der Waals interaction between non-polar CNTs arises from fluctuating dipole moments in carbon atoms (Carel 2006). Under an ideal condition, the interaction was estimated to be 100 to 200 times stronger than the adhesion force that the foot of a gecko can generate (Yurdumakan et al 2005). Since chloride molecules have permanent multipoles, the van der Waals interaction between the polyvinyl chloride substrate film and the CNTs are expected to be even larger than the interaction between CNTs (Carel 2006).

Figure 3 illustrates the experimental setup for stretching the dilute CNT mat. First, the CNT mat $(10 \times 20 \mathrm{~mm})$ was gently transferred onto a transparent film which was then supported by a paper frame $(60 \times 90 \mathrm{~mm})$. Sheet resistance was measured by plotting resistance of the CNT mat at set increments of $5 \mathrm{~mm}$ in the longitudinal axis (stretch direction) from a reference point, interpolating on the resistance value on the resistance line at the distance equal to the width of the CNT mat, and then subtracting contact resistance which is the resistance value of the y-intercept of the resistance line (figure 4). Also visible light transmittance of the CNT-mat/transparent-film composite was measured by ultraviolet-visible spectroscopy (UV-Vis) and transmittance of a pristine transparent film was subtracted to obtain transmittance of the CNT mat. We specifically focused on a wavelength of $550 \mathrm{~nm}$ in our detailed discussion to obtain a direct comparison with other authors (Wu et al 2004; Zhou et al 2006). After the measurement, the CNT-mat/transparent-film composite was fixed to a tabletop clamp and unidirectionally stretched after cutting the paper support at two opposite sides. To hold the film under the stretched condition, both edges of stretched CNT-mat/transparent-film composite was then adhered to a PMMA substrate by epoxy glue and both the sheet resistance and the visible light transmittance were measured again.

The experiments have been conducted under the room temperature (between $20^{\circ} \mathrm{C}$ and $25^{\circ} \mathrm{C}$ ). Solar UAV is expected to operate under the temperature between $-90^{\circ} \mathrm{C}$ and $-45^{\circ} \mathrm{C}$ (MacDonald 2005), and the temperature fluctuation should cause a thermal fatigue. This thermal effect should be evaluated in our future work.

\section{Results and discussions}

The axial strains applied to the CNT mat on a polyvinyl chloride film were 40,60 and $80 \%$ (figure 5), while those for the CNT mat on a polyurethane elastomer film were 40 and 110 (figure 6). Beyond the strains, both substrates started breaking apart. Five specimens were tested in each case and the data obtained for $80 \%$ stretch was shown in table 1 as an example. In figures 5 and 6 , the black curves represent the reference data of CNT films made by vacuum filtration ( $\mathrm{Hu}$ et al 2004) which is typically used to fabricate transparent conducting 

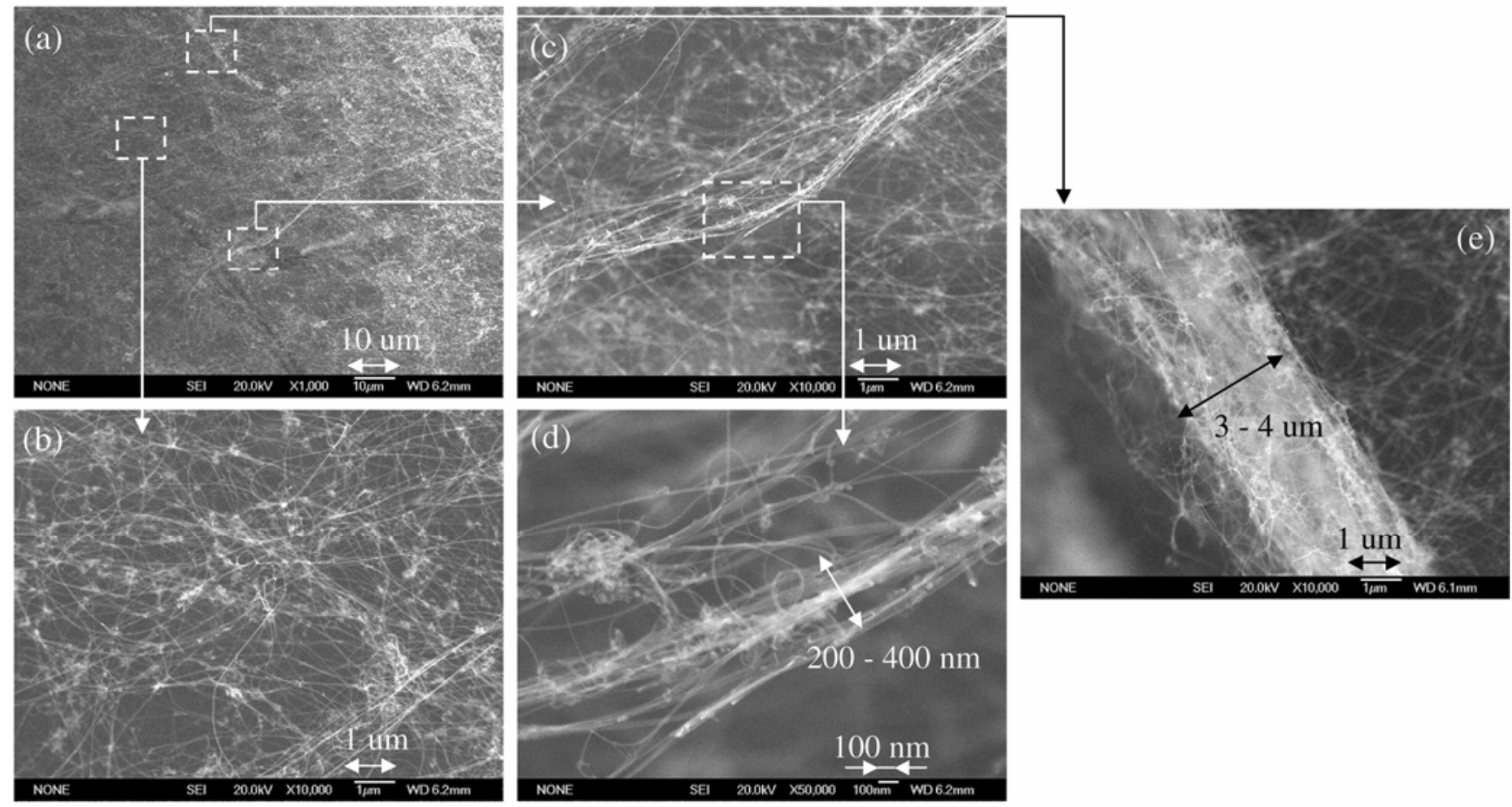

Figure 2. (a) Scanning electron microscopy (SEM) images of a dilute CNT mat $(\times 1000)$, (b) well distributed MWNT network $(\times 10,000)$, (c) bundled MWNTs $(\times 10,000)$, (d) bundled MWNTs $(\times 50,000)$, and $(\mathbf{e})$ MWNTs forming larger bundles $(\times 10,000)$. Bundles are defined as clusters of MWNTs attracted to each other by van der Waals forces. The bundle size is measured to be hundreds of nanometers $(\mathbf{d})$ to a few micrometers $(\mathbf{e})$.
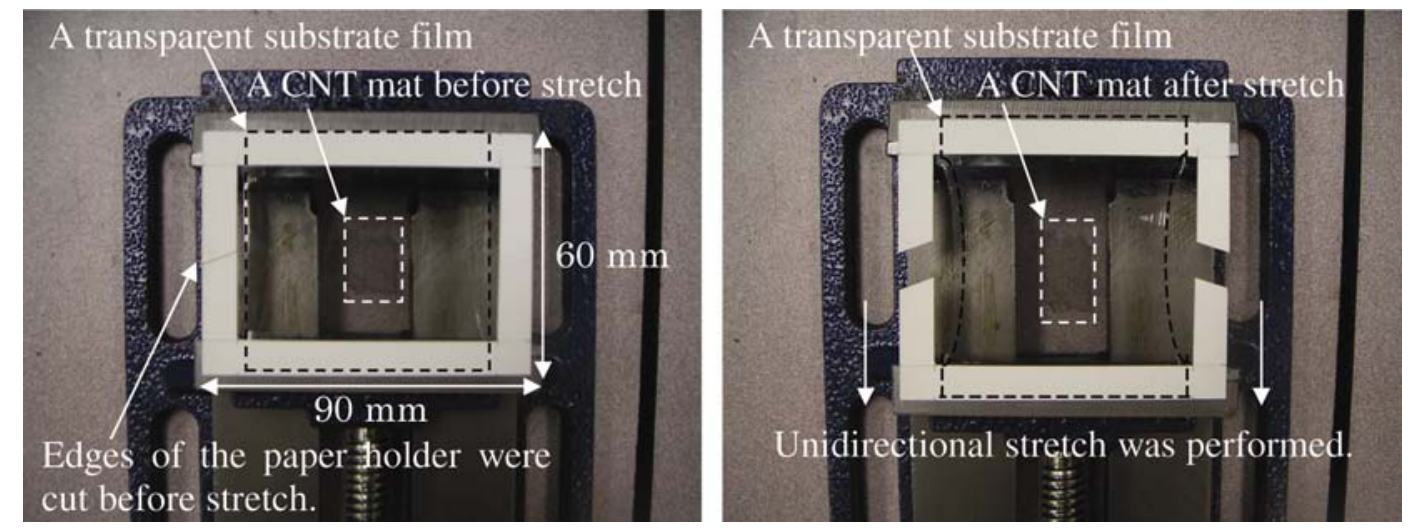

Figure 3. Typical experimental set-up of a dilute CNT mat unidirectional stretch.

CNT films. Figure 7 shows typical visible light transmittance (400-800 nm wavelength) of a dilute CNT mat before and after $80 \%$ strain stretching on a polyvinyl chloride film.

More improvement on both sheet resistance and visible light transmittance was observed when stretch ratio of the dilute-CNT-mat/polyvinyl-chloride-film composite was increased (figure 5). Figure 7 shows that visible light transmittance was improved throughout the wavelength of $400 \mathrm{~nm}$ to $800 \mathrm{~nm}$ after $80 \%$ strain stretching. The images in figure 8 were optical micrographs of a dilute
CNT mat after stretching, showing no fibre breakage of the dilute CNT mat.

On the other hand, the sheet resistance did not decrease when the dilute CNT mat on a polyurethane elastomer film was stretched by $40 \%$ strain (figure 6 ), and the sheet resistance increased when $110 \%$ strain was applied; the optical micrographs in figure 9 shows CNTs starting to break at $40 \%$ strain and large tears appeared at $110 \%$ strain. The observed fractures in the CNT mat naturally increase the sheet resistance although they may improve the transmittance. 
Table 1. Sheet resistance and transmittance at $550 \mathrm{~nm}$ wavelength of a dilute CNT mat before (noted as original) and after (noted as stretched) stretch. Five CNT mat samples on polyvinyl chloride films with no adhesive were stretched by $80 \%$ strain. Average sheet resistance was reduced to $71 \%$ and average transmittance at $550 \mathrm{~nm}$ wavelength was improved by $37 \%$ as compared to the original CNT mat, respectively.

\begin{tabular}{|c|c|c|c|c|}
\hline \multirow[b]{2}{*}{ Sample } & \multicolumn{2}{|c|}{ Sheet resistance $(\Omega / \square)$} & \multicolumn{2}{|c|}{ Transmittance@ @550mm (\%) } \\
\hline & $R_{\mathrm{s} \_ \text {original }}$ & $R_{\text {s_s }_{\text {stretched }}}$ & $T_{\text {original }}$ & $T_{\text {stretched }}$ \\
\hline 1 & 400 & 250 & $59 \cdot 8$ & $79 \cdot 5$ \\
\hline 2 & 320 & 240 & $53 \cdot 5$ & $76 \cdot 4$ \\
\hline 3 & 260 & 175 & $53 \cdot 9$ & $75 \cdot 0$ \\
\hline 4 & 300 & 220 & $58 \cdot 1$ & $78 \cdot 2$ \\
\hline 5 & 230 & 180 & $57 \cdot 1$ & $77 \cdot 1$ \\
\hline Average & 302 & 213 & $56 \cdot 5$ & $77 \cdot 2$ \\
\hline Ratio (stretched/original) & \multicolumn{2}{|c|}{$71 \%$} & \multicolumn{2}{|c|}{$137 \%$} \\
\hline
\end{tabular}

Table 2. Nomenclature.

\begin{tabular}{ll}
\hline Nomenclature & \multicolumn{1}{c}{ Description } \\
\hline$A_{\text {original }}$ & Area of a rectangle arbitrarily taken on the CNT mat before stretching. \\
$A_{\text {stretched }}$ & The new area of $A_{\text {original }}$ after stretching. \\
$L_{\text {original }}$ & Length of a rectangle arbitrarily taken on the CNT mat before stretching. \\
$L_{\text {stretched }}$ & The new length of $L_{\text {original }}$ after stretching. \\
$N_{\text {original }}$ & Density of the CNT mat in the axial cross section normal to the stretch direction (before stretching). \\
$N_{\text {stretched }}$ & Density of the CNT mat in the axial cross section normal to the stretch direction (after stretching). \\
$R_{\text {s_original }}$ & Sheet resistance of the CNT mat (before stretching). \\
$R_{\text {s_stretched }}$ & Sheet resistance of the CNT mat (after stretching). \\
$T_{\text {original }}$ & Visible light transmittance of the CNT mat (before stretching). \\
$T_{\text {stretched }}$ & Visible light transmittance of the CNT mat (after stretching). \\
$W_{\text {original }}$ & Width of a rectangle arbitrarily taken on the CNT mat before stretching. \\
$W_{\text {stretched }}$ & The new width of $W_{\text {original }}$ after stretching. \\
$\varepsilon$ & Longitudinal strain of the CNT mat. \\
$v$ & Poisson's ratio of the CNT mat. This value varies according to the longitudinal strain, $\varepsilon$. \\
\hline
\end{tabular}

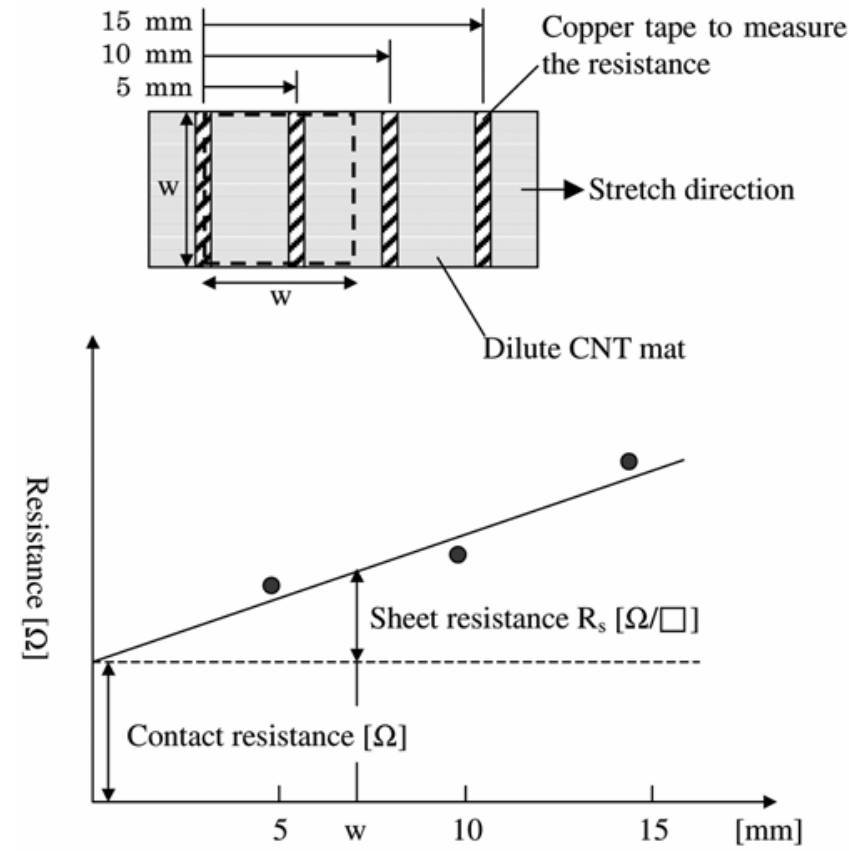

Figure 4. Measurement of a dilute CNT mat sheet resistance.

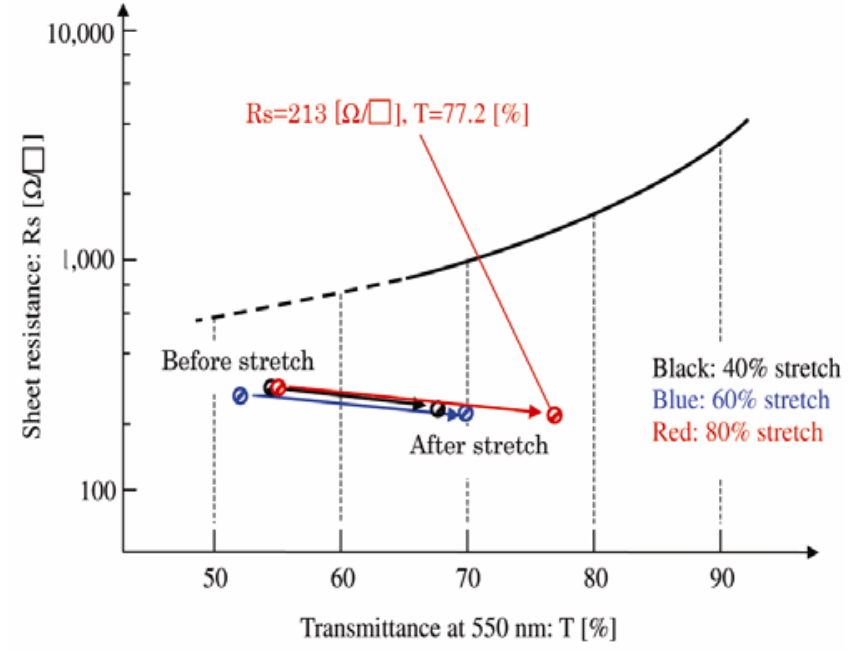

Figure 5. Sheet resistance and transmittance at $550 \mathrm{~nm}$ wavelength of a dilute CNT mat before and after stretch. Five samples were stretched by 40,60 and $80 \%$ strain respectively and average data is shown in each case. A polyvinyl chloride film with no adhesive was used as a substrate. The black curve represented the data of CNT films fabricated by vacuum filtration (Hu et al 2004). 
The presence of an adhesive on the polyurethane elastomer film is assumed to prevent the CNT mat from stretching freely, and the state of strain of the film is too much for the CNT mat to bear. However, when the polyvinyl chloride film is used without any adhesive, the only forces holding the CNT mat onto the film are the van der Waals forces. These forces should allow transverse slippage of CNTs on the film surface and hence no CNTs are broken on the film.

Figure 10 shows optical micrographs of a dilute CNT mat on a polyvinyl chloride film before and after $80 \%$ strain stretch. Dotted rectangles represent the same area of the CNT mat before and after the stretch. As expected, the deformed shape indicates an axial stretching combined with a lateral contraction. The number of pixels inside a rectangle counted on the Adobe Photoshop

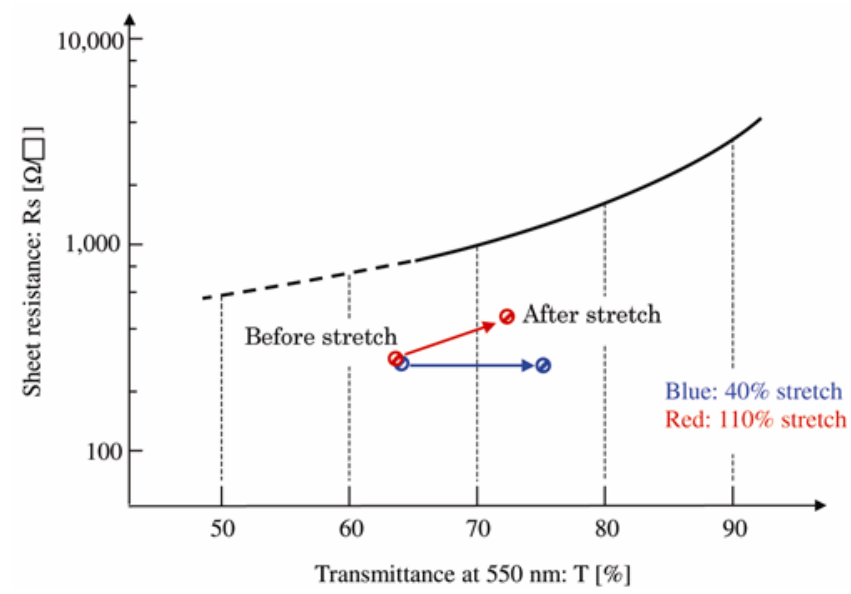

Figure 6. Sheet resistance and transmittance at $550 \mathrm{~nm}$ wavelength of a dilute CNT mat before and after stretch. Five samples were stretched by 40 and $110 \%$ strain respectively and average data is shown in each case. A polyurethane elastomer film with adhesive was used as a substrate. The black curve represented the data of CNT films fabricated by vacuum filtration (Hu et al 2004).

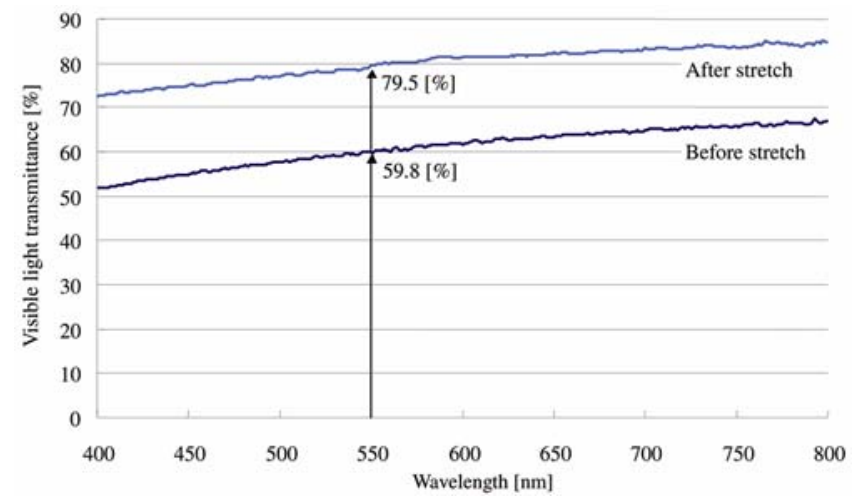

Figure 7. Typical visible light transmittance (400-800 nm wavelengths) of a dilute CNT mat before and after $80 \%$ stretch when a polyvinyl chloride film was used to support the CNT mat
(Adobe Inc., San Jose CA) increased after stretch from 92,232 to 131,920 , indicating that the area of the initial rectangle had increased. Also, after stretch, the mean value of luminosity increased from 64.21 to 77.13 , in agreement with the observed improvement of transmittance at $550 \mathrm{~nm}$ wavelength (figure 5). Luminosity indicates the brightness of micrographs: 0 when it is completely black and 200 when it is completely white. Note that a big black area on the left side of each picture is a part of a black marker used to identify the location of the targeted area after stretch.

Figures 11 and 12 illustrate the predicted sheet resistance and visible light transmittance at $550 \mathrm{~nm}$ wavelength, respectively, as functions of stretch strain. Also shown for comparison in the figures are the experimental data of CNT mat stretched on the polyvinyl chloride substrate films. The predictions were based on the assumed deformation as schematically shown in figure 13. Nomenclatures used for the predictions are summarized in table 2.

Modelling of the dilute CNT mat sheet resistance after stretch was performed as follows. As the area changes under a given stretch, the CNTs inside the area will remain the same. The sheet resistance measured in the stretch direction can be assumed to be inversely proportional to the CNT density in the axial cross section normal to the stretch direction. Therefore, by referring to figure 13, sheet resistance of a stretched CNT mat over sheet resistance of an original CNT mat, $R_{\text {s_stretched }_{\text {}}}$ $R_{\text {s_original }}$ can be described as follows.

$$
\begin{aligned}
R_{\mathrm{s}_{-} \text {stretched }} / R_{\mathrm{s}_{-} \text {original }} & =N_{\text {original }} / N_{\text {stretched }} \\
& =N_{\text {original }} /\left[1 /(1-v \varepsilon) N_{\text {original }}\right] \\
& =1-v \varepsilon .
\end{aligned}
$$

Poisson's ratio, $v$, of the CNT mat could be obtained by comparing a longitudinal and lateral length of a box arbitrarily taken in optical micrographs before stretching ( $\left.L_{\text {original }}, W_{\text {original }}\right)$ and those of the same box after stretching ( $\left.L_{\text {stretched, }} W_{\text {stretched }}\right)$ (see figure 10$)$.

The visible light transmittance may be predicted using similar reasoning. Suppose the transmittance was proportional to the area not covered by CNTs. Then the area of a stretched CNT mat over area of an original CNT mat, $A_{\text {stretched }} / A_{\text {original }}$ can be described as follows:

$$
A_{\text {stretched }} / A_{\text {original }}=(1+\varepsilon)(1-v \varepsilon) .
$$

The resulting transmittance is then given by

$$
T_{\text {stretched }} / T_{\text {original }}=(1+\varepsilon)(1-v \varepsilon) .
$$

Experimental results showed slightly better improvement than predictions after stretch for sheet resistance (figure 11). Nevertheless, these simple prediction models capture 


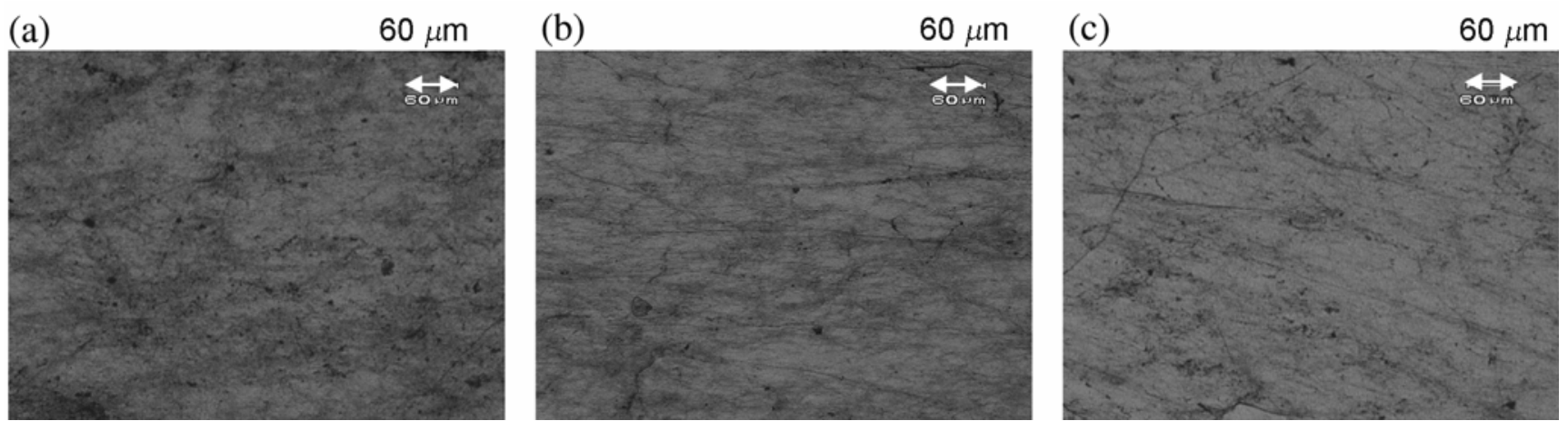

Figure 8. Optical micrographs of a dilute CNT mat on a polyvinyl chloride film (no adhesive) after stretch: (a) $40 \%$ strain, (b) $60 \%$ strain, and (c) $80 \%$ strain. No breakage of the dilute CNT mat was observed.
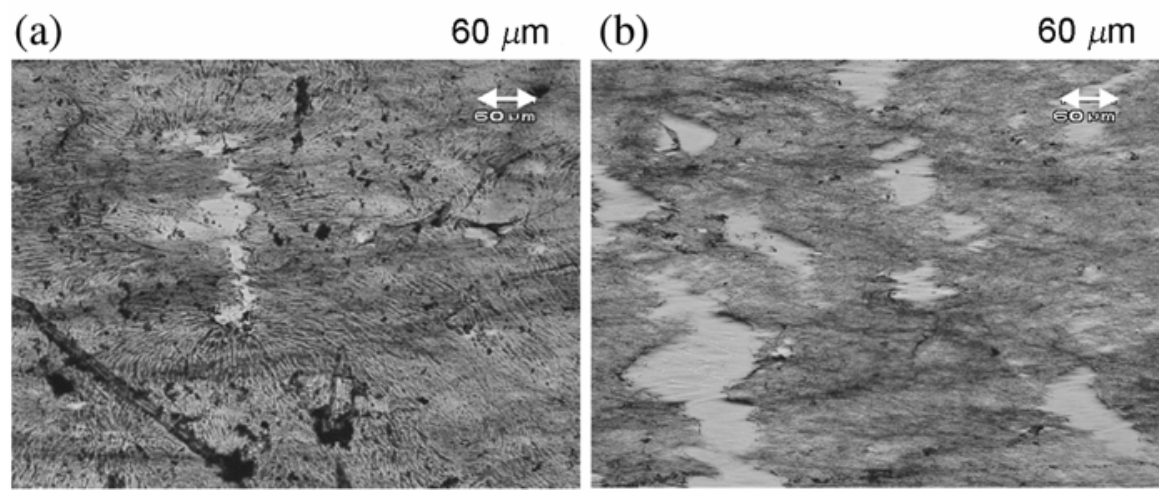

Figure 9. Optical micrographs of a dilute CNT mat on a polyurethane elastomer (with an adhesive) after stretch: (a) 40\% strain, and (b) $110 \%$ strain. Mild fractures (a) and severe fractures (b) of the dilute CNT mat were observed.
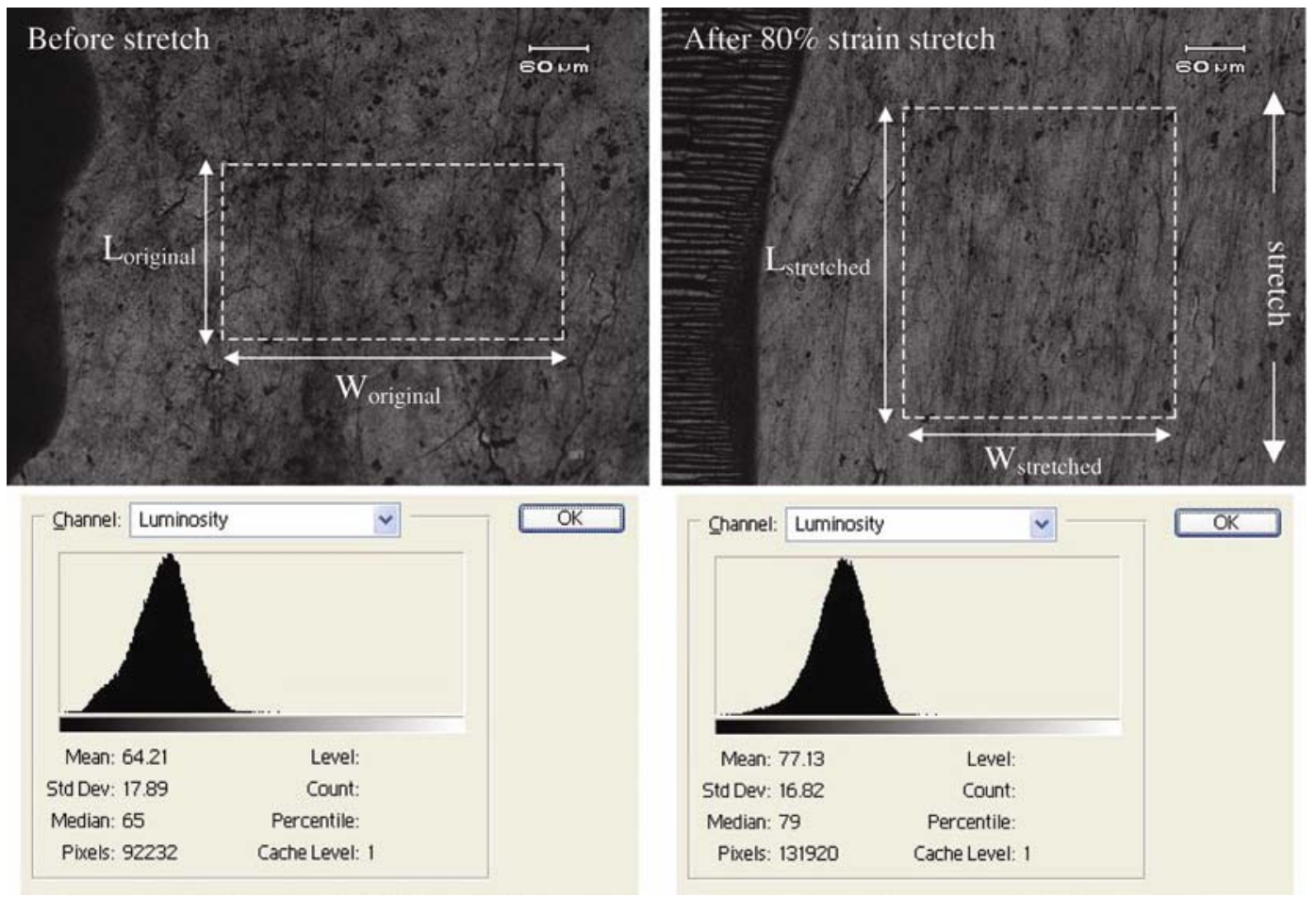

Figure 10. Optical micrographs of a dilute CNT mat on a polyvinyl chloride film (no adhesive) before and after $80 \%$ strain stretch. After stretching, mean luminosity increased from $64 \cdot 21$ to $77 \cdot 13$. 


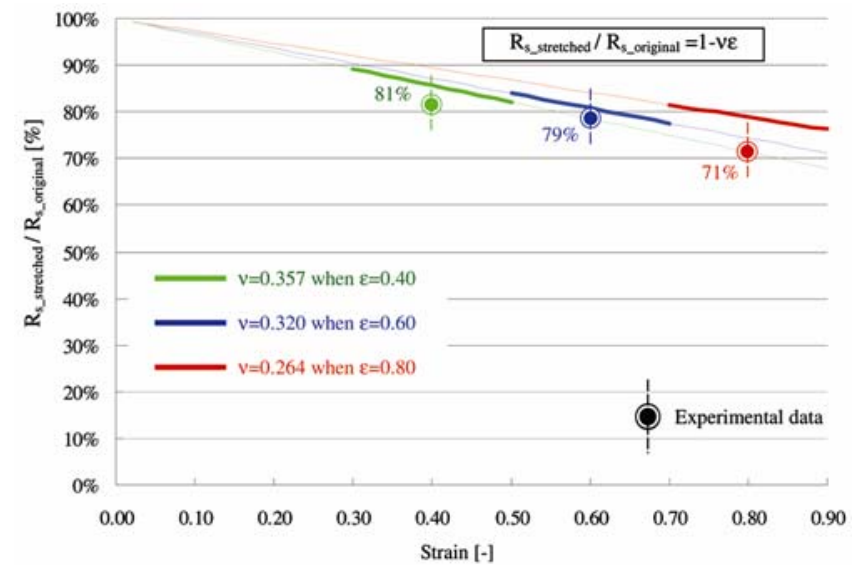

Figure 11. A curve modeling a dilute CNT mat sheet resistance after stretch with plotted experimental data when a polyvinyl chloride film was used as a substrate.

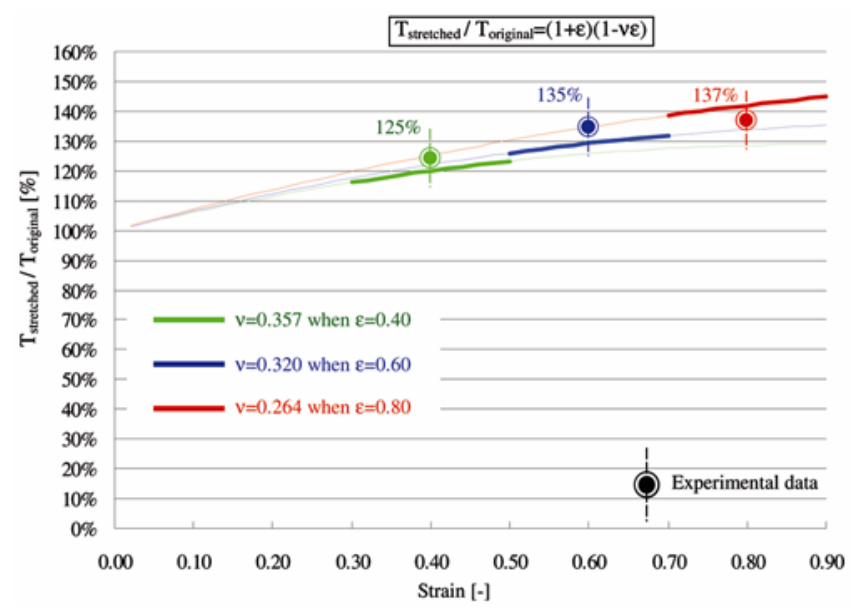

Figure 12. A curve modeling a dilute CNT mat transmittance at $550 \mathrm{~nm}$ wavelength after stretch with plotted experimental data when a polyvinyl chloride film was used as a substrate.

the main mechanisms of sheet resistance and visible light transmittance changes induced by stretching of the CNT mat.

\section{Conclusions}

Unidirectional stretching was applied to improve the optical and electrical properties of a dilute CNT mat. After the dilute CNT mat on a polyvinyl chloride film was stretched by $80 \%$ strain, it reached the average sheet resistance of $213 \Omega / \square$ and the average transmittance at $550 \mathrm{~nm}$ wavelength of $77 \cdot 2 \%$ (see figure 5 and table 1 ). The transmittance value does not subtract the value of PET film, so when the stretched CNT mat is transplanted onto a PET substrate, its transmittance at $550 \mathrm{~nm}$ wave-

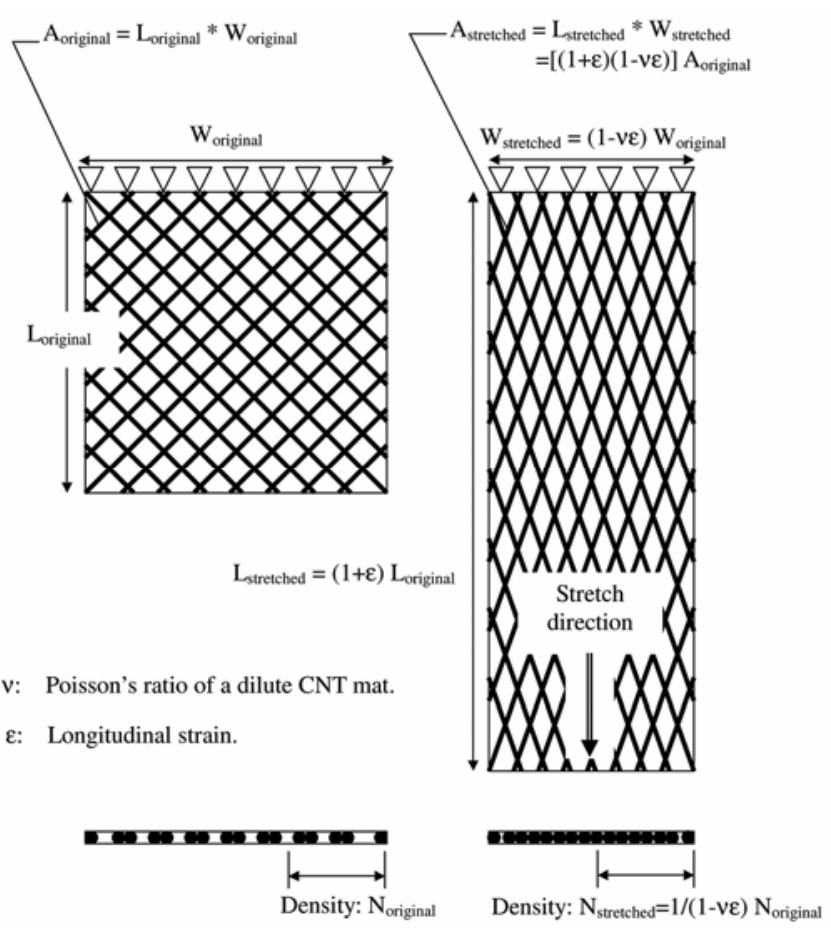

Figure 13. A model representing the dilute CNT mat behaviour before and after stretching.

length should get smaller than that of commercially available ITO film on PET substrate, that is $80 \%$ with sheet resistance of $200 \Omega / \square$ (Zhou et al 2006). However, once a substrate film which can withstand more than $80 \%$ of stretch is obtained in our approach, the optical and electrical properties can potentially be improved further.

As seen in figures 1 and 2 , our dilute CNT mat contains bundles. CNTs inside the bundle have less contribution to the electrical conductivity than CNTs on the bundle surface, and thus increase the overall resistance (Zhou et al 2006). In that sense, a chemical approach to obtain a dilute CNT mat with more evenly distributed CNT networks and fewer bundles should be pursued as future work. Once achieved, combined with the stretching method to improve the optical and electrical properties discussed in this paper, the dilute CNT mat is expected to meet the requirement as a crack-resistant transparent conducting film for use as solar-UAV power supplies.

\section{Acknowledgements}

The present paper is based on work supported by the Air Force Office of Scientific Research through a MURI grant FA9550-06-1-0326 to the University of Washington with Dr. B. Les Lee as Program Manager. Appreciation is extended to the NSF Materials Creation Training Program (DGE-0654431) for the use of experimental facilities. 


\section{References}

Cairns D R et al 2005 IEEE 931451

Carel J O 2006 Interfacial forces in aqueous media (CRC Press: Boca Raton, FL 33487) Ch. 2, 2nd edn

Grace D et al 2001 Broadband communications from high altitude platforms-the HeliNet Solution, Wireless Personal Mobile Conference

Hu L et al 2004 Nano Letts. 42513

Iijima S 1991 Nature 35456
Lashmore D et al 2007 United States Patent Application Publication no. US 2007/0036709 A1

MacDonald A E 2005 A global profiling system for improved weather and climate prediction (American Meteorological Society Article)

Teets Jr E H et al 2002 Meteorological support of the helios world record high altitude flight to 96,863 feet (NASA report TM-2002-210727)

Wu Z et al 2004 Science 3051273

Yurdumakan B et al 2005 Chem. Commun. 3799

Zhang M et al 2005 Science 3091215

Zhou Y et al 2006 Appl. Phys. Lett. 88123109 